\title{
On certain products of Banach algebras with applications to harmonic analysis
}

\author{
by \\ Mehdi Sangani Monfared (Windsor)
}

\begin{abstract}
Given Banach algebras $A$ and $B$ with spectrum $\sigma(B) \neq \emptyset$, and given $\theta \in$ $\sigma(B)$, we define a product $A \times_{\theta} B$, which is a strongly splitting Banach algebra extension of $B$ by $A$. We obtain characterizations of bounded approximate identities, spectrum, topological center, minimal idempotents, and study the ideal structure of these products. By assuming $B$ to be a Banach algebra in $\mathcal{C}_{0}(X)$ whose spectrum can be identified with $X$, we apply our results to harmonic analysis, and study the question of spectral synthesis, and primary ideals.
\end{abstract}

1. Introduction and preliminaries. The products $A \times_{\theta} B$ of $\mathrm{Ba}$ nach algebras defined in this paper (Definition 2.1) were first introduced by T. Lau [18] for a special class of Banach algebras that are pre-duals of von Neumann algebras, and for which the identity of the dual is a multiplicative linear functional. In this paper we show that these products can be defined for Banach algebras in a fairly general setting. The resulting products can be viewed as strongly splitting Banach algebra extensions.

The study of these products has significance in two respects. First, the products exhibit many properties that are not shared in general by arbitrary strongly splitting extensions. As a simple example, the algebra of upper triangular matrices is a direct sum of diagonal matrices with strictly upper triangular matrices. While both direct summands are commutative, the algebra itself is not commutative. This is in contrast with the case of the extensions $A \times{ }_{\theta} B$ (Proposition 2.3). Second, the products $A \times{ }_{\theta} B$ can serve as a source of examples (or counterexamples) for various purposes in functional and harmonic analysis. In this respect, they have found applications in the study of character amenability, which is the subject of a forthcoming paper [21].

2000 Mathematics Subject Classification: Primary 46H20, 46H10, 46J20, 43A45; Secondary 46J10, 43A30.

Key words and phrases: Banach algebras, extensions, ideals, minimal idempotents, cyclic amenability, topological center, spectral synthesis.

This work was supported by the NSERC grant number 298449 . 
We recall that the spectrum $\sigma(B)$ of a Banach algebra $B$ is the set of all non-zero multiplicative linear functionals on $B$. If $A$ is a commutative, semisimple Banach algebra, it can be viewed as a Banach algebra in $\mathcal{C}_{0}(\sigma(A))$ via the Gelfand transformation $a \mapsto \widehat{a}$. We say that $A$ is regular Tauberian if $\widehat{A}=\{\widehat{a}: a \in A\}$ is regular (that is, separates points from closed sets) and its elements with compact support are dense in $\widehat{A}$. The kernel of a closed set $E \subset \sigma(A)$ is defined by $k(E)=\{a \in A: \phi(a)=$ 0 for all $\phi \in E\}$. The hull of a set $I \subset A$ is defined by $h(I)=\{\phi \in$ $\sigma(A): \phi(a)=0$ for all $a \in I\}$. A closed set $E \subset \sigma(A)$ is called a spectral set of $A$ if $k(E)$ is the only closed ideal of $A$ whose hull is equal to $E$. Equivalently, $E$ is a spectral set if for each $a \in A$ such that $\widehat{a}(E)=\{0\}$, and each $\varepsilon>0$, there exists $b \in A$ such that $\widehat{b}$ has compact support disjoint from $E$ and $\|a-b\|<\varepsilon$. $E$ is called a local spectral set if the conditions in the definition hold under the additional assumption that $a$ has compact support. We say that spectral synthesis holds in $A$ if every closed subset of $\sigma(A)$ is a spectral set [13, Sec. 39]. We denote by $k_{00}(E)$ the set of all $b \in A$ such that $\widehat{b}$ has compact support disjoint from $E$.

Let $X$ be a locally compact Hausdorff space and $B$ a regular subalgebra of $\mathcal{C}_{0}(X)$. Suppose that $B$ is a Banach algebra with respect to a certain norm $\|\cdot\|$. For each $x \in X$, let $\epsilon_{x}$ denote the evaluation functional at $x$. Then the map $x \mapsto \epsilon_{x}$ is a homeomorphism from $X$ into $\sigma(B)$. If this map is surjective, we say $B$ is a Banach algebra in $\mathcal{C}_{0}(X)$, where $X$ is the spectrum of $B$. For simplicity, if $x_{0} \in X$ we write $I_{x_{0}}$ in place of $k\left(\left\{\epsilon_{x_{0}}\right\}\right)$, in other words,

$$
I_{x_{0}}=k\left(\left\{\epsilon_{x_{0}}\right\}\right)=\left\{u \in B: u\left(x_{0}\right)=0\right\} .
$$

For a locally compact group $G$, the Fourier algebra $A(G)$ consists of all coefficients of the left regular representation of $G[9]$. The norm of $A(G)$ is defined by regarding it as the continuous linear functionals on the group $C^{*}$-algebra, $C^{*}(G)$. In other words, for $u \in A(G)$,

$$
\|u\|=\sup _{f \in L^{1}(G),\|f\|_{\Sigma} \leq 1}\left|\int_{G} f(x) u(x) d x\right|,
$$

where $\Sigma$ denotes the collection of all equivalence classes of continuous unitary representations of $G$. When $G$ is commutative, $A(G) \cong L^{1}(\widehat{G})$ via the Fourier transformation. The spectrum of $A(G)$ consists of all point evaluation functionals $\left\{\epsilon_{x}: x \in G\right\}$, and can be identified with $G$ in a natural way. For every $x \in G,\{x\}$ is a set of spectral synthesis for $A(G)$. It is well known that $A(G)$ has a bounded approximate identity if and only if $G$ is amenable. 
Recall that the dual of a Banach algebra $A$ carries a natural left and right $A$-module structure defined by

$$
\langle a \cdot \phi, b\rangle=\langle\phi, b a\rangle, \quad\langle\phi \cdot a, b\rangle=\langle\phi, a b\rangle \quad\left(a, b \in A, \phi \in A^{\prime}\right) .
$$

The first Arens product $\square$ on $A^{\prime \prime}$ is then defined by the following relations:

$$
\langle\Phi \square \Psi, \phi\rangle=\langle\Phi, \Psi \odot \phi\rangle, \quad\langle\Psi \odot \phi, a\rangle=\langle\Psi, \phi \cdot a\rangle .
$$

Similarly, the second Arens product $\diamond$ on $A^{\prime \prime}$ is defined by

$$
\langle\Phi \diamond \Psi, \phi\rangle=\langle\Psi, \phi \odot \Phi\rangle, \quad\langle\phi \odot \Phi, a\rangle=\langle\Phi, a \cdot \phi\rangle .
$$

The (first) topological center $\mathfrak{Z}_{\mathrm{t}}\left(A^{\prime \prime}\right)$ of $A^{\prime \prime}$ is the set of all $\Phi \in A^{\prime \prime}$ such that

$$
\Phi \square \Psi=\Phi \diamond \Psi \quad \text { for all } \Psi \in A^{\prime \prime} .
$$

In general, the topological center is a closed subalgebra of $\left(A^{\prime \prime}, \square\right)$ containing $A$. The algebra $A$ is called Arens regular (respectively, strongly Arens irregular) if $\mathfrak{Z}_{\mathrm{t}}\left(A^{\prime \prime}\right)=A^{\prime \prime}$ (respectively, $\mathfrak{Z}_{\mathrm{t}}\left(A^{\prime \prime}\right)=A$ ). The notion of "strongly Arens irregular" was introduced recently by Dales and Lau in [5].

A Banach algebra $A$ is called amenable (respectively, contractible) if for every Banach $A$-bimodule $E$, every continuous derivation $d: A \rightarrow E^{\prime}$ (respectively, $d: A \rightarrow E$ ) is an inner derivation [14]. $A$ is called weakly amenable if every continuous derivation $d: A \rightarrow A^{\prime}$ is an inner derivation. A derivation $d: A \rightarrow A^{\prime}$ is called cyclic if

$$
\langle d(a), b\rangle+\langle d(b), a\rangle=0 \quad \text { for all } a, b \in A .
$$

If every continuous cyclic derivation from $A$ to $A^{\prime}$ is inner, then $A$ is called cyclic amenable [11].

2. Definition and structural properties. Let $A$ and $B$ be Banach algebras with $\sigma(B) \neq \emptyset$, and let $\phi, \psi \in \sigma(B)$. If we equip the set $A \times B$ with the usual $\mathbb{C}$-module structure, then the multiplication

$$
(a, b) \cdot\left(a^{\prime}, b^{\prime}\right)=\left(a a^{\prime}+\phi(b) a^{\prime}+\psi\left(b^{\prime}\right) a, b b^{\prime}\right)
$$

turns $A \times B$ into a non-associative algebra. One can then verify that if $A$ has non-trivial multiplication, a necessary and sufficient condition for associativity of the multiplication is that $\phi=\psi$. Since in this paper we deal only with associative algebras, we are led to the following definition.

2.1. Definition. Let $A$ and $B$ be Banach algebras with $\sigma(B) \neq \emptyset$, and let $\theta \in \sigma(B)$. The $\theta$-Lau product $A \times{ }_{\theta} B$ is defined as the Cartesian product $A \times B$ with the algebra multiplication

$$
(a, b) \cdot\left(a^{\prime}, b^{\prime}\right)=\left(a a^{\prime}+\theta(b) a^{\prime}+\theta\left(b^{\prime}\right) a, b b^{\prime}\right),
$$

and with the norm $\|(a, b)\|=\|a\|+\|b\|$.

2.2. Remarks. (a) The space $A \times_{\theta} B$ is a Banach algebra. If we allow $\theta=0$, we obtain the usual direct product of Banach algebras. Since direct 
products often exhibit different properties, we have excluded the possibility that $\theta=0$. If $B=\mathbb{C}$ and $\theta: \mathbb{C} \rightarrow \mathbb{C}$ is the identity map, then $A \times{ }_{\theta} \mathbb{C}$ coincides with the unitization of $A$.

(b) In $A \times_{\theta} B$ we identify $A \times\{0\}$ with $A$, and $\{0\} \times B$ with $B$. Then $A$ is a closed ideal while $B$ is a closed subalgebra of $A \times_{\theta} B$, and

$$
A \times_{\theta} B / A \cong B \quad \text { (isometric isomorphism). }
$$

In other words, $A \times_{\theta} B$ is a strongly splitting Banach algebra extension of $B$ by $A$. We note that the multiplication of $A \times_{\theta} B$ is not induced from a two-cocycle $T \in \mathcal{Z}^{2}(B, A)$, as the extension $A \times_{\theta} B$ is not singular unless $A$ has trivial multiplication (for more on extensions of Banach algebras see [1], [4]).

2.3. Proposition. Let $A$ and $B$ be a Banach algebras and $\theta \in \sigma(B)$.

(i) Given Banach algebras $\mathcal{A}, \mathcal{B}$, and given $\phi \in \sigma(\mathcal{B})$, the Banach algebras $A \times_{\theta} B$ and $\mathcal{A} \times_{\phi} \mathcal{B}$ are isomorphic if and only if there exist Banach algebra isomorphisms $\Psi: A \rightarrow \mathcal{A}$ and $\Phi: B \rightarrow \mathcal{B}$ such that $\theta=\phi \circ \Phi$.

(ii) $A \times_{\theta} B$ is commutative if and only if both $A$ and $B$ are commutative.

(iii) $\left(a_{0}, b_{0}\right)$ is an identity for $A \times_{\theta} B$ if and only if $a_{0}=0$ and $b_{0}=1_{B}$ is an identity for $B$.

(iv) $\left(\left(a_{\alpha}, b_{\alpha}\right)\right)_{\alpha}$ is a bounded left (right, or two-sided) approximate identity for $A \times_{\theta} B$ if and only if $\left\|a_{\alpha}\right\| \rightarrow 0$ and $\left(b_{\alpha}\right)_{\alpha}$ is a bounded left (right, or two-sided) approximate identity for $B$. A similar statement is true for unbounded approximate identities.

Proof. For briefness we only give the proof for (iv). First assume that $\left(\left(a_{\alpha}, b_{\alpha}\right)\right)_{\alpha}$ is a bounded left approximate identity. Thus there exists $M>0$ such that $\left\|b_{\alpha}\right\| \leq\left\|a_{\alpha}\right\|+\left\|b_{\alpha}\right\| \leq M$ for all $\alpha$. Also for every $b \in B$ we must have

$$
\left\|\left(a_{\alpha}, b_{\alpha}\right)(0, b)-(0, b)\right\|=|\theta(b)|\left\|a_{\alpha}\right\|+\left\|b_{\alpha} b-b\right\| \rightarrow 0 .
$$

Since $b$ is arbitrary we conclude that $\left\|a_{\alpha}\right\| \rightarrow 0$, and $\left(b_{\alpha}\right)_{\alpha}$ is a bounded left approximate identity for $B$.

Conversely, if $\left\|a_{\alpha}\right\| \rightarrow 0$ and $\left(b_{\alpha}\right)_{\alpha}$ is a bounded left approximate identity for $B$, then the net $\left(a_{\alpha}, b_{\alpha}\right)_{\alpha}$ is bounded, and for every $(a, b) \in A \times_{\theta} B$, $\left\|\left(a_{\alpha}, b_{\alpha}\right)(a, b)-(a, b)\right\| \leq\left\|a_{\alpha}\right\|\|a\|+\|a\|\left|\theta\left(b_{\alpha}\right)-1\right|+|\theta(b)|\left\|a_{\alpha}\right\|+\left\|b_{\alpha} b-b\right\| \rightarrow 0$, since $\theta\left(b_{\alpha}\right) \rightarrow 1$.

The dual of the space $A \times{ }_{\theta} B$ can be identified with $A^{\prime} \times B^{\prime}$ in the natural way $\langle(\phi, \psi),(a, b)\rangle=\phi(a)+\psi(b)$. The dual norm on $A^{\prime} \times B^{\prime}$ is of course the maximum norm $\|(\phi, \psi)\|=\max \{\|\phi\|,\|\psi\|\}$. We recall that on $A^{\prime} \times B^{\prime}$ the weak* topology coincides with the product of the weak ${ }^{*}$ topologies of $A^{\prime}$ and $B^{\prime}[17,(17.14)$, p. 161]. The following result identifies the spectrum 
of $A \times{ }_{\theta} B$. We also provide a description of various neighborhood bases in $\sigma\left(A \times{ }_{\theta} B\right)$ for our later purposes. Note that the topology of $\sigma\left(A \times{ }_{\theta} B\right)$ is the induced weak* topology from $A^{\prime} \times B^{\prime}$.

Part (i) of the following result is a generalization of a result proved by Lau [18, Proposition 3.6, p. 166]

2.4. Proposition. Let $A$ and $B$ be Banach algebras with $\sigma(B) \neq \emptyset$. Let $\theta \in \sigma(B)$ and

$$
E=\{(\phi, \theta): \phi \in \sigma(A)\}, \quad F=\{(0, \psi): \psi \in \sigma(B)\} .
$$

Set $E=\emptyset$ if $\sigma(A)=\emptyset$. Then

(i) $\sigma\left(A \times{ }_{\theta} B\right)=E \cup F$.

(ii) In $\sigma\left(A \times \times_{\theta} B\right), F$ is closed, while $E \cup\{(0, \theta)\}$ is compact.

(iii) All sets of the form $U \times\{\theta\}$, where $U$ is a neighborhood of $\phi$ in $\sigma(A)$, form a neighborhood base at $(\phi, \theta)$. If $\psi \neq \theta$, all sets of the form $\{0\} \times W$, where $W$ is a neighborhood of $\psi$ in $\sigma(B)$ not containing $\theta$, form a neighborhood base at $(0, \psi)$. Finally, all sets of the form

$$
(U \cap \sigma(A)) \times\{\theta\} \cup\{0\} \times W,
$$

where $U$ is a neighborhood of 0 in $A^{\prime}$ and $W$ is a neighborhood of $\theta$ in $\sigma(B)$, form a neighborhood base at $(0, \theta)$.

Proof. (i) We prove the inclusion $\sigma\left(A \times{ }_{\theta} B\right) \subset E \cup F$, the reverse inclusion is easy to verify directly. Suppose that $(\phi, \psi) \in \sigma\left(A \times_{\theta} B\right)$. Then for every $(a, b),\left(a^{\prime}, b^{\prime}\right) \in A \times_{\theta} B$ we have

$$
\left\langle(\phi, \psi),\left(a a^{\prime}+\theta(b) a^{\prime}+\theta\left(b^{\prime}\right) a, b b^{\prime}\right)\right\rangle=(\phi(a)+\psi(b))\left(\phi\left(a^{\prime}\right)+\psi\left(b^{\prime}\right)\right),
$$

which implies

$$
\begin{aligned}
\phi\left(a a^{\prime}\right)+\theta(b) \phi\left(a^{\prime}\right)+\theta\left(b^{\prime}\right) \phi(a)+\psi\left(b b^{\prime}\right) & \\
& =\phi(a) \phi\left(a^{\prime}\right)+\phi(a) \psi\left(b^{\prime}\right)+\psi(b) \phi\left(a^{\prime}\right)+\psi(b) \psi\left(b^{\prime}\right) .
\end{aligned}
$$

If we take $b=b^{\prime}=0$, it follows that $\phi\left(a a^{\prime}\right)=\phi(a) \phi\left(a^{\prime}\right)$ for all $a, a^{\prime} \in A$, and hence $\phi \in \sigma(A) \cup\{0\}$. Next, if we take $a=a^{\prime}=0$, it follows that $\psi \in \sigma(B) \cup\{0\}$. But $\psi=0$ implies that $\phi=0$, which is impossible since $(\phi, \psi) \neq(0,0)$. So we have shown that $\psi \in \sigma(B)$. Now if $\phi=0$, then $(\phi, \psi)=(0, \psi) \in F$, which is what we want. If $\phi \neq 0$, we can rewrite the above equation as

$$
(\theta(b)-\psi(b)) \phi\left(a^{\prime}\right)+\left(\theta\left(b^{\prime}\right)-\psi\left(b^{\prime}\right)\right) \phi(a)=0 .
$$

Choosing $a^{\prime}=0$, and $a$ such that $\phi(a) \neq 0$, it follows that $\psi\left(b^{\prime}\right)=\theta\left(b^{\prime}\right)$ for all $b^{\prime} \in B$, and hence $\psi=\theta$. This means that $(\phi, \psi)=(\phi, \theta) \in E$. Thus $\sigma\left(A \times_{\theta} B\right) \subset E \cup F$.

The proofs of (ii) and (iii) are left for the reader. 
2.5. Note. For the rest of this paper, we shall use the letters $E$ and $F$ exclusively to denote the sets defined in (2).

The following two results which are stated for left ideals are also true for right or two-sided ideals.

2.6. Proposition. Let $A$ and $B$ be Banach algebras and $\theta \in \sigma(B)$. Suppose that $I$ is a left ideal of $A, J$ is a left ideal of $B$, and $M=I \times J$.

(i) If $J \subset \operatorname{ker} \theta$, then $M$ is a left ideal in $A \times_{\theta} B$.

(ii) If $J \not \subset \operatorname{ker} \theta$, then $M$ is a left ideal in $A \times_{\theta} B$ if and only if $I=A$.

The proof is routine and is omitted.

Next we consider the converse. Suppose that $M$ is a left ideal of $A \times{ }_{\theta} B$ and

$$
\begin{aligned}
& I=\{a \in A:(a, b) \in M \text { for some } b \in B\}, \\
& J=\{b \in B:(a, b) \in M \text { for some } a \in A\} .
\end{aligned}
$$

The question is whether in general $I$ and $J$ are ideals and whether $M=I \times J$. We will show by examples that in general we can neither expect $I$ to be an ideal, nor to have $M=I \times J$ (even if $A$ is unital). However we have the following result.

2.7. Proposition. Let $M, I$, and $J$ be as above. Then

(i) $J$ is a left ideal in $B$.

(ii) If $J \subset \operatorname{ker} \theta$, then $I$ is a left ideal of $A$. Furthermore if $A$ has a left approximate identity and if $M$ is closed, then $M=I \times J$.

(iii) If $J \not \subset \operatorname{ker} \theta$ but $I$ is a left ideal of $A$, then $I=A$.

Proof. (i) is easy.

For (ii) suppose that $J \subset \operatorname{ker} \theta$, and let $a \in I$. Then $(a, b) \in M$ for some $b \in J$. Then for every $a^{\prime} \in A$,

$$
\left(a^{\prime}, 0\right)(a, b)=\left(a^{\prime} a+\theta(b) a^{\prime}, 0\right)=\left(a^{\prime} a, 0\right) \in M .
$$

Thus $a^{\prime} a \in I$, that is, $I$ is a left ideal of $A$.

Next let $\left(a_{\alpha}\right)_{\alpha}$ be a left approximate identity (not necessarily bounded) for $A$, and suppose that $a_{0} \in I, b_{0} \in J$. We will show that $\left(a_{0}, b_{0}\right) \in M$, which proves $M=I \times J$. Let $b \in J$ and $a \in I$ be such that $\left(a_{0}, b\right),\left(a, b_{0}\right) \in M$. Then $\left(a_{\alpha}, 0\right)\left(a_{0}, b\right)=\left(a_{\alpha} a_{0}, 0\right) \in M$, and

$$
\left\|\left(a_{\alpha} a_{0}, 0\right)-\left(a_{0}, 0\right)\right\|=\left\|a_{\alpha} a_{0}-a_{0}\right\| \rightarrow 0 .
$$

Since $M$ is closed it follows that $\left(a_{0}, 0\right) \in M$. Similarly, we can show that $(a, 0) \in M$. Consequently, $\left(a, b_{0}\right)-(a, 0)=\left(0, b_{0}\right) \in M$, and hence

$$
\left(a_{0}, 0\right)+\left(0, b_{0}\right)=\left(a_{0}, b_{0}\right) \in M .
$$


(iii) Suppose that $b_{0} \in J-\operatorname{ker} \theta$. Then $\left(a_{0}, b_{0}\right) \in M$ for some $a_{0} \in I$. Thus for every $a \in A$,

$$
(a, 0)\left(a_{0}, b_{0}\right)=\left(a a_{0}+\theta\left(b_{0}\right) a, 0\right) \in M .
$$

Hence $a \in I$ as $a a_{0} \in I$ and $\theta\left(b_{0}\right) \neq 0$. That is, $I=A$.

2.8. Examples. The following two examples serve to show that the condition $J \subset \operatorname{ker} \theta$ is necessary in the second part of Proposition 2.7.

Let $B$ be a regular Banach algebra in $\mathcal{C}_{0}(X)$, where $X$ is the spectrum of $B$. Let $x_{0} \in X, \theta=\epsilon_{x_{0}}$, and consider the product $B \times{ }_{\theta} B$.

(a) Let $K$ be a proper compact subset of $X$ containing $x_{0}$ and at least one other element. Define

$$
\begin{aligned}
I & =\left\{u \in B:\left.u\right|_{K} \text { is a constant function }\right\}, \\
M & =\left\{(u, v) \in B \times{ }_{\theta} B: u \in I, v\left(x_{0}\right)=-u\left(x_{0}\right)\right\} .
\end{aligned}
$$

Regularity of $B$ implies that $I \neq \emptyset$. If $J$ is defined as in (4), then $J=B \not \subset$ $\operatorname{ker} \theta$. It is easy to check that $M$ is a closed ideal of $B \times_{\theta} B$, while neither $I$ is an ideal of $B$, nor $M=I \times J$.

(b) We modify the above example, by defining $M$ to be

$$
M=\left\{(u, v) \in B \times \times_{\theta} B: v\left(x_{0}\right)=-u\left(x_{0}\right)\right\} .
$$

In this case $M$ is closed ideal, and $I=J=B$ (that is, both $I$ and $J$ are ideals), but nonetheless $M \neq I \times J$.

2.9. Remark. There does not seem to be an easy description for various radicals of $A \times{ }_{\theta} B$. However if $\mathfrak{R}$ denotes either strong, or Jacobson, or prime radical, and if $\mathfrak{R}(B)=\{0\}$, then

$$
\mathfrak{R}\left(A \times_{\theta} B\right)=\mathfrak{R}(A) .
$$

It is easy to characterize the idempotent elements of $A \times_{\theta} B$. In the following, we would like to characterize the minimal idempotents of these algebras because of their importance in the ideal theory of $A \times_{\theta} B$. Suppose that $B$ is an algebra. We recall that a non-zero element $\mu \in B$ is called a minimal idempotent if $\mu^{2}=\mu$ and $\mu B \mu=\mathbb{C} \mu$. It is well known that when $B$ is semiprime, $L$ is a minimal left (respectively, right) ideal of $B$ if and only if $L=B \mu$ (respectively, $L=\mu B$ ), where $\mu$ is a minimal idempotent in $B$ [3, Proposition 6 , p. 155]. In the group algebra $L^{1}(G)$ of a locally compact group $G$, the minimal idempotents are the coefficients of integrable representations of $G[2]$. For the Fourier algebra $A(G)$, minimal idempotents exist if and only if $G$ is discrete, in which case the minimal idempotents are given by characteristic functions $\chi_{\{x\}}$ of single point sets [7, Theorem 5.5, p. 360].

2.10. TheOREM. An element $\left(a_{0}, b_{0}\right)$ is a minimal idempotent of $A \times{ }_{\theta} B$ if and only if one of the following conditions are satisfied: 
(i) $b_{0}=0$ and $a_{0}$ is a minimal idempotent of $A$.

(ii) $a_{0}=0, \theta\left(b_{0}\right)=0$, and $b_{0}$ is a minimal idempotent of $B$.

(iii) $b_{0}$ is a minimal idempotent such that $b_{0} b b_{0}=\theta(b) b_{0}$ for all $b \in B$, $\theta\left(b_{0}\right)=1$, and $a_{0}$ satisfies

$$
\left\{\begin{array}{l}
a_{0}^{2}=-a_{0}, \\
a_{0} a a_{0}+a a_{0}+a_{0} a+a=0 \text { for all } a \in A .
\end{array}\right.
$$

Proof. The sufficiency part of the theorem is left for the reader. We prove the necessity. Let $\left(a_{0}, b_{0}\right)$ be a minimal idempotent; we show at least one of the above three conditions holds.

The condition $\left(a_{0}, b_{0}\right)^{2}=\left(a_{0}, b_{0}\right)$ implies that

$$
\begin{gathered}
b_{0}^{2}=b_{0}, \\
a_{0}^{2}+2 \theta\left(b_{0}\right) a_{0}=a_{0} .
\end{gathered}
$$

If $(a, b) \in A \times{ }_{\theta} B$ is arbitrary, there must exist $\lambda_{a, b} \in \mathbb{C}$ such that

$$
\left(a_{0}, b_{0}\right)(a, b)\left(a_{0}, b_{0}\right)=\lambda_{a, b}\left(a_{0}, b_{0}\right),
$$

which is equivalent to

$$
\begin{gathered}
a_{0} a a_{0}+\theta\left(b_{0}\right)\left(a a_{0}+a_{0} a\right)+\theta(b) a_{0}^{2}+\theta\left(b_{0}\right)^{2} a+2 \theta(b) \theta\left(b_{0}\right) a_{0}=\lambda_{a, b} a_{0} \\
b_{0} b b_{0}=\lambda_{a, b} b_{0} .
\end{gathered}
$$

Comparing (6) and (9) it follows that either $b_{0}=0$, or $b_{0}$ is a minimal idempotent of $B$ with $\lambda_{a, b}=\lambda_{0, b}$ for all $a \in A$.

If we assume $b_{0}=0$, then (7) implies $a_{0}^{2}=a_{0}$, and (8) implies

$$
a_{0} a a_{0}+\theta(b) a_{0}^{2}=\lambda_{a, b} a_{0} \text { for all }(a, b) .
$$

Upon substituting $b=0$, we obtain $a_{0} a a_{0}=\lambda_{a, 0} a_{0}$, for all $a \in A$. Thus $a_{0}$ is a minimal idempotent, proving condition (i).

Alternatively if we assume $b_{0} \neq 0$ is a minimal idempotent, and $\lambda_{a, b}=$ $\lambda_{0, b}$ for all $a$, then either $\theta\left(b_{0}\right)=0$ or $\theta\left(b_{0}\right)=1$. We consider two cases.

CASE I: $\theta\left(b_{0}\right)=0$. Then (7) and (8) imply

$$
\begin{aligned}
a_{0}^{2} & =a_{0}, \\
a_{0} a a_{0}+\theta(b) a & =\lambda_{0, b} a_{0} \quad \text { for all } a, b .
\end{aligned}
$$

Substituting $a=0$ in the last equation implies $\lambda_{0, b} a_{0}=0$ for all $b \in B$, which can be true only if $a_{0}=0$ (the alternative $\lambda_{0, b}=0$ also leads to $a_{0}=0$ if we substitute $a=a_{0}$, and $b=0$ in (11)), thus proving (ii).

CASE II: $\theta\left(b_{0}\right)=1$. Then (7) implies $a_{0}^{2}=-a_{0}$, and consequently upon substituting $b=0$ in (8) we get

$$
a_{0} a a_{0}+a a_{0}+a_{0} a+a=\lambda_{0,0} a_{0} \text { for all } a .
$$


If we set $a=0$ in the above equation we conclude $\lambda_{0,0}=0$, and therefore

$$
a_{0} a a_{0}+a a_{0}+a_{0} a+a=0 \quad \text { for all } a .
$$

Finally, from (9) and the fact that $\theta\left(b_{0}\right)=1$ we get $\theta(b)=\lambda_{0, b}$ for all $b \in B$, hence proving (iii).

We remark that the conditions in $(*)$ do not imply that $a$ is an idempotent of $A$. For example when $A$ has an identity $e_{A}$, the only element of $A$ satisfying $(*)$ is $-e_{A}$.

Next we turn our attention to the question of amenability and topological center of $A \times{ }_{\theta} B$.

Amenability has well known hereditary properties [14, 4, 20]. In particular, since $A \times{ }_{\theta} B$ is a strongly splitting extension of $B$, it is amenable (respectively, contractible) if and only if both $A$ and $B$ are amenable (respectively, contractible).

With regard to weak amenability, although weak amenability of $A$ and $B$ implies the weak amenability of $A \times_{\theta} B$, the converse is not true in general [15]. However, we can state the following theorem.

2.11. Theorem. Weak amenability of $A \times_{\theta} B$ implies the weak amenability of $B$ and cyclic amenability of $A$.

Proof. The weak amenability of $B$ follows from a general result about Banach algebra extensions proved in [19, Lemma 2.3, p. 183]. It remains to prove the cyclic amenability of $A$. Let $P: A \times_{\theta} B \rightarrow A$ be the projection on $A$, and let $d: A \rightarrow A^{\prime}$ be a continuous cyclic derivation. Then we prove

$$
D={ }^{t} P \circ d \circ P: A \times{ }_{\theta} B \rightarrow A^{\prime} \times B^{\prime}
$$

is a continuous derivation. In fact, for every $(a, b),\left(a^{\prime}, b^{\prime}\right)$, and $(c, e)$ in $A \times{ }_{\theta} B$, we have

$$
\begin{aligned}
\left\langle D\left((a, b)\left(a^{\prime}, b^{\prime}\right)\right),(c, e)\right\rangle & =\left\langle d\left(a a^{\prime}+\theta(b) a^{\prime}+\theta\left(b^{\prime}\right) a\right), c\right\rangle \\
& =\left\langle d\left(a a^{\prime}\right)+\theta(b) d\left(a^{\prime}\right)+\theta\left(b^{\prime}\right) d(a), c\right\rangle .
\end{aligned}
$$

On the other hand,

$$
\begin{aligned}
\left\langle D(a, b) \cdot\left(a^{\prime}, b^{\prime}\right),(c, e)\right\rangle & =\left\langle d(a), a^{\prime} c+\theta\left(b^{\prime}\right) c+\theta(e) a^{\prime}\right\rangle \\
& =\left\langle d(a) \cdot a^{\prime}+\theta\left(b^{\prime}\right) d(a), c\right\rangle+\left\langle d(a)\left(a^{\prime}\right) \theta, e\right\rangle,
\end{aligned}
$$

and

$$
\begin{aligned}
\left\langle(a, b) \cdot D\left(a^{\prime}, b^{\prime}\right),(c, e)\right\rangle & =\left\langle d\left(a^{\prime}\right), c a+\theta(e) a+\theta(b) c\right\rangle \\
& =\left\langle a \cdot d\left(a^{\prime}\right)+\theta(b) d\left(a^{\prime}\right), c\right\rangle+\left\langle d\left(a^{\prime}\right)(a) \theta, e\right\rangle .
\end{aligned}
$$

Adding (15) to (16) and comparing with (14) we conclude that $D$ is a derivation since $d(a)\left(a^{\prime}\right)+d\left(a^{\prime}\right)(a)=0$. From the weak amenability of $A \times{ }_{\theta} B$ it follows that $D=\delta_{(\phi, \psi)}$ for some $(\phi, \psi) \in A^{\prime} \times B^{\prime}$. We claim that $d=\delta_{\phi}$; 
indeed, for all $a, a^{\prime} \in A$,

$$
\begin{aligned}
\left\langle d(a), a^{\prime}\right\rangle & =\left\langle{ }^{t} P \circ d \circ P(a, 0),\left(a^{\prime}, 0\right)\right\rangle \\
& =\left\langle(a, 0) \cdot(\phi, \psi)-(\phi, \psi) \cdot(a, 0),\left(a^{\prime}, 0\right)\right\rangle \\
& =\left\langle\phi, a^{\prime} a\right\rangle-\left\langle\phi, a a^{\prime}\right\rangle \\
& =\left\langle a \cdot \phi-\phi \cdot a, a^{\prime}\right\rangle,
\end{aligned}
$$

as we wanted to show.

To state our next result we note that if $B$ is a Banach algebra and $\theta \in \sigma(B)$, then $\theta \in \sigma\left(B^{\prime \prime}\right)$ in a natural way, when $B^{\prime \prime}$ is equipped with either of its Arens products.

2.12. Proposition. Let $A$ and $B$ be Banach algebras and $\theta \in \sigma(B)$. Suppose that $A^{\prime \prime}, B^{\prime \prime}$, and $\left(A \times_{\theta} B\right)^{\prime \prime}$ are equipped with their first (or second) Arens products. Then

$$
\left(A \times_{\theta} B\right)^{\prime \prime} \cong A^{\prime \prime} \times_{\theta} B^{\prime \prime} \quad \text { (isometric isomorphism). }
$$

Proof. Assume that $A^{\prime \prime}, B^{\prime \prime}$, and $\left(A \times_{\theta} B\right)^{\prime \prime}$ are equipped with the first Arens product. The multiplication in $A^{\prime \prime} \times_{\theta} B^{\prime \prime}$ is given by

$$
(\Phi, \Psi)\left(\Phi^{\prime}, \Psi^{\prime}\right)=\left(\Phi \square \Phi^{\prime}+\Psi(\theta) \Phi^{\prime}+\Psi^{\prime}(\theta) \Phi, \Psi \square \Psi^{\prime}\right) .
$$

To compute the first Arens product in $\left(A \times_{\theta} B\right)^{\prime \prime}$, let $(a, b) \in A \times_{\theta} B$, $(\phi, \psi) \in A^{\prime} \times B^{\prime}$, and $(\Phi, \Psi),\left(\Phi^{\prime}, \Psi^{\prime}\right) \in A^{\prime \prime} \times B^{\prime \prime}$ be arbitrary. Then one can calculate, respectively,

$$
\begin{aligned}
(\phi, \psi) \cdot(a, b) & =(\phi \cdot a+\theta(b) \phi, \psi \cdot b+\phi(a) \theta), \\
\left(\Phi^{\prime}, \Psi^{\prime}\right) \odot(\phi, \psi) & =\left(\Phi^{\prime} \odot \phi+\Psi^{\prime}(\theta) \phi, \Phi^{\prime}(\phi) \theta+\Psi^{\prime} \odot \psi\right), \\
(\Phi, \Psi) \square\left(\Phi^{\prime}, \Psi^{\prime}\right) & =\left(\Phi \square \Phi^{\prime}+\Psi(\theta) \Phi^{\prime}+\Psi^{\prime}(\theta) \Phi, \Psi \square \Psi^{\prime}\right) .
\end{aligned}
$$

Comparing (17) with (18) shows that the identity mapping is an isometric isomorphism between $\left(A \times_{\theta} B\right)^{\prime \prime}$ and $A^{\prime \prime} \times_{\theta} B^{\prime \prime}$.

Calculations with the second Arens product are similar. On one hand, we have

$$
(\Phi, \Psi)\left(\Phi^{\prime}, \Psi^{\prime}\right)=\left(\Phi \diamond \Phi^{\prime}+\Psi(\theta) \Phi^{\prime}+\Psi^{\prime}(\theta) \Phi, \Psi \diamond \Psi^{\prime}\right) ;
$$

and on the other hand,

$$
\begin{aligned}
(a, b) \cdot(\phi, \psi) & =(a \cdot \phi+\theta(b) \phi, b \cdot \psi+\phi(a) \theta), \\
(\phi, \psi) \odot(\Phi, \Psi) & =(\phi \odot \Phi+\Psi(\theta) \phi, \Phi(\phi) \theta+\psi \odot \Psi), \\
(\Phi, \Psi) \diamond\left(\Phi^{\prime}, \Psi^{\prime}\right) & =\left(\Phi \diamond \Phi^{\prime}+\Psi(\theta) \Phi^{\prime}+\Psi^{\prime}(\theta) \Phi, \Psi \diamond \Psi^{\prime}\right) .
\end{aligned}
$$

2.13. Corollary. $\mathfrak{Z}_{\mathrm{t}}\left[\left(A \times_{\theta} B\right)^{\prime \prime}\right]=\mathfrak{Z}_{\mathrm{t}}\left(A^{\prime \prime}\right) \times_{\theta} \mathfrak{Z}_{\mathrm{t}}\left(B^{\prime \prime}\right)$. In particular, $A \times_{\theta} B$ is Arens regular (respectively, strongly Arens irregular) if and only if both $A$ and $B$ are Arens regular (respectively, strongly Arens irregular). 
3. Spectral synthesis. We start this section with the following result which will be used throughout.

3.1. TheOREM. Let $A$ and $B$ be commutative Banach algebras, and let $\theta \in \sigma(B)$.

(i) $A \times_{\theta} B$ is semisimple if and only if both $A$ and $B$ are semisimple.

(ii) If $A$ and $B$ are semisimple, $b \in B$ and its Gelfand transform $\widehat{b}$ has compact support, then for every $a \in A,(a, b)^{\wedge}=(\widehat{a}, \widehat{b})$ has compact support.

(iii) If $A$ and $B$ are semisimple, then $A \times_{\theta} B$ is regular if and only if both $A$ and $B$ are regular. If in addition $B$ is Tauberian, then so is $A \times{ }_{\theta} B$.

Proof. (i) Suppose that $A$ and $B$ are semisimple. Using the characterization of spectrum in Proposition 2.4, we verify that the Gelfand map is injective; in fact, if $(a, b)^{\wedge}=(\widehat{a}, \widehat{b})=(0,0)$ then

$$
\begin{aligned}
& \langle(\widehat{a}, \widehat{b}),(\phi, \theta)\rangle=\phi(a)+\theta(b)=0 \quad(\phi \in \sigma(A)), \\
& \langle(\widehat{a}, \widehat{b}),(0, \psi)\rangle=\psi(b)=0 \quad(\psi \in \sigma(B)) \text {. }
\end{aligned}
$$

This implies that $a=b=0$. The converse can be proved similarly.

(ii) Assume that $b \in B$ and $\operatorname{supp} \widehat{b}$ is compact in $\sigma(B)$. Then for every $a \in A$,

$$
\operatorname{supp}(\widehat{a}, \widehat{b}) \subset E \cup\{(0, \theta)\} \cup\{(0, \psi): \psi \in \operatorname{supp} \widehat{b}\}
$$

since by Proposition 2.4 the set on the right hand side is compact (hence closed) and $(\widehat{a}, \widehat{b})$ is zero outside this set, proving that $(\widehat{a}, \widehat{b})$ has compact support.

(iii) Suppose that both $A$ and $B$ are regular. Let $S \subset \sigma\left(A \times_{\theta} B\right)$ be closed and $\left(\phi_{0}, \psi_{0}\right) \in \sigma\left(A \times_{\theta} B\right)-S$. Define

$$
\begin{aligned}
& S_{1}=S \cap F, \quad S_{2}=S \cap E, \\
& S_{1}^{\prime}=\left\{\psi \in \sigma(B):(0, \psi) \in S_{1}\right\}, \quad S_{2}^{\prime}=\left\{\phi \in \sigma(A):(\phi, \theta) \in S_{2}\right\} .
\end{aligned}
$$

The sets $S_{1}^{\prime}$ and $S_{2}^{\prime}$ are closed subsets of $\sigma(B)$ and $\sigma(A)$, respectively. To separate $\left(\phi_{0}, \psi_{0}\right)$ from $S$ we consider several cases:

CAsE I: $\left(\phi_{0}, \psi_{0}\right)=(0, \theta)$. In this case 0 is not in the closure of $S_{2}^{\prime}$ in $\sigma(A) \cup\{0\}$, and hence $S_{2}^{\prime}$ is compact in $\sigma(A)$. Regularity of $A$ implies that there exists $a_{0} \in A$ such that

$$
\left\langle\widehat{a}_{0}, \phi\right\rangle=\phi\left(a_{0}\right)=-1 \quad \text { for all } \phi \in S_{2}^{\prime},
$$

[13, Corollary 39.16, p. 493]. Next using regularity of $B$, we choose $b_{0} \in B$ 
such that $\widehat{b}_{0}(\theta)=1$ and $\widehat{b}_{0}\left(S_{1}^{\prime}\right)=\{0\}$. Then

$$
\begin{aligned}
& \left\langle\left(\widehat{a}_{0}, \widehat{b}_{0}\right),(0, \theta)\right\rangle=\widehat{b}_{0}(\theta)=1, \\
& \left\langle\left(\widehat{a}_{0}, \widehat{b}_{0}\right),(0, \psi)\right\rangle=\widehat{b}_{0}(\psi)=0 \quad \text { for all } \psi \in S_{1}^{\prime}, \\
& \left\langle\left(\widehat{a}_{0}, \widehat{b}_{0}\right),(\phi, \theta)\right\rangle=\widehat{a}_{0}(\phi)+\widehat{b}_{0}(\theta)=-1+1=0 \quad \text { for all } \phi \in S_{2}^{\prime} .
\end{aligned}
$$

This means that $\left(\widehat{a}_{0}, \widehat{b}_{0}\right)$ separates $(0, \theta)$ from $S$.

CASE II: $\left(\phi_{0}, \psi_{0}\right)=\left(0, \psi_{0}\right)\left(\psi_{0} \neq \theta\right)$. Using regularity of $B$, choose $b_{0} \in B$ such that

$$
\widehat{b}_{0}\left(\psi_{0}\right)=1, \quad \widehat{b}_{0}\left(S_{1}^{\prime} \cup\{\theta\}\right)=\{0\} ;
$$

then of course $\left(0, \widehat{b}_{0}\right)$ separates $\left(0, \psi_{0}\right)$ from $S$.

CASE III: $\left(\phi_{0}, \psi_{0}\right)=\left(\phi_{0}, \theta\right)$. In this case $\phi_{0} \notin S_{2}^{\prime}$. From regularity of $A$ there exists $a_{0} \in A$ such that $\widehat{a}_{0}\left(\phi_{0}\right)=1$ and $\widehat{a}_{0}(\phi)=0$ for all $\phi \in S_{2}^{\prime}$. Now $\left(\widehat{a}_{0}, 0\right)$ separates $\left(\phi_{0}, \theta\right)$ from $S$.

The proofs of the rest of the statements in (iii) are left for the reader.

As a consequence of the abstract Tauberian theorem [13, Theorem 39.27, p. 499] and the above result we obtain:

3.2. Corollary. Let $A$ and $B$ be commutative, semisimple, regular Banach algebras, and suppose that $B$ is Tauberian. Then the empty set $\emptyset$ is a spectral set of $A \times_{\theta} B$. In particular, every proper closed ideal in $A \times_{\theta} B$ is contained in a maximal modular ideal.

For the rest of this section we assume that $A$ and $B$ are commutative, semisimple, regular Banach algebras. We also recall our standing assumption that $E$ and $F$ are used exclusively to denote

$$
E=\{(\phi, \theta): \phi \in \sigma(A)\}, \quad F=\{(0, \psi): \psi \in \sigma(B)\} .
$$

It follows from [13, Theorem 39.19, p. 494] that if $K \subset \sigma(B)$ is a nonspectral set for $B$, then $\{(0, \psi): \psi \in K\}$ is a non-spectral set for $A \times{ }_{\theta} B$.

We show in the next result that even a spectral set of $B$ may no longer be a spectral set for the extension $A \times_{\theta} B$.

3.3. Proposition. Let $A$ and $B$ be commutative, semisimple, regular Banach algebras, and suppose the empty set is not a spectral set for $A$. Then the singleton $\{(0, \theta)\}$ is not a spectral set for $A \times{ }_{\theta} B$.

Proof. By our assumption, $A$ has a proper closed ideal $I$ such that $h(I)$ $=\emptyset$. Let $J=\operatorname{ker} \theta=\{b \in B: \theta(b)=0\}$, and $M=I \times J$. By Proposition 2.6, $M$ is a closed ideal of $A \times_{\theta} B$. If $(0, \psi) \in h(M)$, then $\psi(b)=0$ for all $b \in \operatorname{ker} \theta$, and therefore by regularity of $B, \psi=\theta$. That is, $h(M) \cap F=$ $\{(0, \theta)\}$. If however $(\phi, \theta) \in h(M)$, then by definition of $M$, we must have $\phi(a)=0$ for all $a \in I$, that is, $\phi \in h(I)$, which is impossible. This means 
that $h(M) \cap E=\emptyset$. Thus $h(M)=\{(0, \theta)\}$. Clearly $M \neq k(\{(0, \theta)\})=A \times J$. Thus $\{(0, \theta)\}$ is not a spectral set.

As a consequence of the above result we can show Ditkin's property is not preserved under Banach algebra extensions. We recall the following definition [13, p. 497].

Let $A$ be a commutative semisimple Banach algebra. We say $A$ satisfies Ditkin's condition if the following conditions hold.

(a) If $a \in A$ and $\phi \in \sigma(A)$ is such that $\widehat{a}(\phi)=0$, then there exists a sequence $\left(b_{n}\right)$ in $A$ such that each $\widehat{b}_{n}$ vanishes in some neighborhood of $\phi$ (depending on $b_{n}$ ) and $\left\|a b_{n}-a\right\| \rightarrow 0$.

(b) If $\sigma(A)$ is non-compact, then for every $a \in A$ there exists a sequence $\left(b_{n}\right)$ in $A$ such that each $\widehat{b}_{n}$ has compact support and $\left\|a b_{n}-a\right\| \rightarrow 0$.

Ditkin's condition has been studied for various algebras by many authors. For example, in the context of the Fourier algebra $A(G)$, it has been studied by Derighetti, Forrest, Kaniuth, Lau and the author [6, 10, 16, 22]. In particular it is known that $A(G)$ (or in general, $A_{p}(G)$ ) satisfies Ditkin's condition if $G$ is amenable [22, Proposition 3.9, p. 420].

3.4. Corollary. Let $A$ and $B$ be commutative, semisimple, regular Banach algebras, and suppose the empty set is not a spectral set for $A$. Then $A \times_{\theta} B$ does not satisfy Ditkin's condition.

Proof. By [13, Corollary 39.26, p. 498] if $A \times{ }_{\theta} B$ satisfies Ditkin's condition and if $S$ is a closed subset of $\sigma\left(A \times{ }_{\theta} B\right)$ whose boundary contains no non-empty perfect set, then $S$ is a spectral set; in particular, $\{(0, \theta)\}$ is a spectral set. This of course contradicts the above proposition.

In our next theorem we assume $B=A(G)$ is the Fourier algebra of a locally compact group $G$, and characterize the circumstances under which singletons are spectral sets in the extensions $A \times_{\theta} B$. It seems that our result cannot be extended to a general commutative, semisimple, regular Banach algebra $B$, unless some restrictive conditions are assumed. For this reason we have decided to state our result for the case of the Fourier algebra, which is of particular interest in harmonic analysis. We recall that $A(G)$ is regular, Tauberian and every singleton $\{x\}$ in $G$ is a spectral set of $A(G)$ [8].

Without loss of generality, we assume $\theta=\epsilon_{e} \in \sigma(A(G))$, where $e$ is the identity of $G$.

3.5. TheOREM. Let $A$ be a commutative, semisimple, regular Banach algebra with identity.

(i) If every singleton $\{\phi\}$ is a spectral set for $A$, then every singleton $\{(\phi, \psi)\}$ is a spectral set for $A \times_{\theta} A(G)$. 
(ii) If every singleton $\{(\phi, \psi)\}$ is a local spectral set for $A \times_{\theta} A(G)$ then every singleton $\{\phi\}$ is a spectral set for $A$.

Therefore every singleton $\{(\phi, \psi)\}$ is a spectral set for $A \times_{\theta} A(G)$ if and only if every singleton $\{\phi\}$ is a spectral set for $A$.

3.6. Remark. We note that as $A$ is assumed to have identity, there is no distinction between spectral sets and local spectral sets in $\sigma(A)$. Statement (ii) means that the converse of (i) is true under a weaker assumption (since $A \times{ }_{\theta} A(G)$ may not have an identity even if $A$ has one-Proposition 2.3).

Proof of Theorem 3.5. (i) CASE I: $(\phi, \psi)=\left(0, \epsilon_{x_{0}}\right), x_{0} \neq e$. Let $(a, u)^{\wedge}=$ $(\widehat{a}, \widehat{u})$ be such that

$$
\left\langle(\widehat{a}, \widehat{u}),\left(0, \epsilon_{x_{0}}\right)\right\rangle=u\left(x_{0}\right)=0 .
$$

Since $\left\{x_{0}\right\}$ is a spectral set for $A(G)$ there exists a sequence $\left(u_{n}\right)$, each $u_{n}$ with compact support $K_{n}$ disjoint from $\left\{x_{0}\right\}$, such that $\left\|u_{n}-u\right\| \rightarrow 0$. It follows that $\left(\widehat{a}, \widehat{u}_{n}\right)$ converges in norm to $(\widehat{a}, \widehat{u})$. Furthermore we have

$$
\operatorname{supp}\left(\widehat{a}, \widehat{u}_{n}\right) \subset E \cup\left\{\left(0, \epsilon_{e}\right\} \cup K_{n}^{\prime}\right.
$$

where $K_{n}^{\prime}=\left\{\left(0, \epsilon_{x}\right): x \in K_{n}\right\}$ is itself a compact subset of $\sigma\left(A \times_{\theta} A(G)\right)$. Thus $\operatorname{supp}\left(\widehat{a}, \widehat{u}_{n}\right)$ is a compact set disjoint from $\left\{\left(0, \epsilon_{x_{0}}\right)\right\}$.

CASE II: $(\phi, \psi)=\left(0, \epsilon_{e}\right)$. This is similar to the previous case if we replace $x$ with $e$ in Case I, except in showing that the support of $\left(\widehat{a}, \widehat{u}_{n}\right)$ is compact we need to use the assumption that $A$ has identity. In fact we can write

$$
\operatorname{supp}\left(\widehat{a}, \widehat{u}_{n}\right)=\overline{\left\{\left(\phi, \epsilon_{e}\right): \phi(a) \neq 0\right\}} \cup \overline{\left\{\left(0, \epsilon_{x}\right): u_{n}(x) \neq 0\right\}} \subset E \cup K_{n}^{\prime},
$$

where the closures are in $\sigma\left(A \times_{\theta} A(G)\right)$, and the last inclusion follows since $A$ is unital and $E=\sigma(A) \times\left\{\epsilon_{e}\right\}$ is compact in $\sigma\left(A \times_{\theta} A(G)\right)$. Of course as before $K_{n}^{\prime}$ is compact and disjoint from $\left\{\left(0, \epsilon_{e}\right)\right\}$, and so is the support of $\left(\widehat{a}, \widehat{u}_{n}\right)$.

CASE III: $(\phi, \psi)=\left(\phi_{0}, \epsilon_{e}\right)$ for some $\phi_{0} \in \sigma(A)$. Let $\left(\widehat{a}_{0}, \widehat{u}_{0}\right)$ be such that

$$
\left\langle\left(\widehat{a}_{0}, \widehat{u}_{0}\right),\left(\phi_{0}, \epsilon_{e}\right\rangle=\phi_{0}\left(a_{0}\right)+u_{0}(e)=0 .\right.
$$

We want to approximate $\left(\widehat{a}_{0}, \widehat{u}_{0}\right)$ with a sequence $\left(\widehat{a}_{n}, \widehat{u}_{n}\right)$ such that each term has a compact support disjoint from $\left\{\left(\phi_{0}, \epsilon_{e}\right)\right\}$. Suppose that $u_{0}(e) \neq 0$ (the other alternative can be handled in a similar fashion). Since both $\left\{\phi_{0}\right\}$ and $\{e\}$ are spectral sets, we can find two sequences $\left(\widehat{a}_{n}\right),\left(\widehat{u}_{n}\right)$ with compact supports in $\sigma(A)$ and in $\sigma(A(G))$ respectively, and two sequences of open neighborhoods, $\left(W_{n}\right)$ and $\left(V_{n}\right)$ of $\phi_{0}$ and $\epsilon_{e}$ respectively, such that

$$
\begin{aligned}
\left\|\widehat{a}_{0}-\widehat{a}_{n}\right\| & \rightarrow 0,\left.\quad \widehat{a}_{n}\right|_{W_{n}}=-u_{0}(e), \\
\left\|\widehat{u}_{0}-\widehat{u}_{n}\right\| & \rightarrow 0,\left.\quad \widehat{u}_{n}\right|_{V_{n}}=u_{0}(e) .
\end{aligned}
$$

Thus over the neighborhood $W_{n} \times\left\{\epsilon_{e}\right\}$ of $\left(\phi_{0}, \epsilon_{e}\right)$ we have $\left.\left(\widehat{a}_{n}, \widehat{u}_{n}\right)\right|_{W_{n} \times\left\{\epsilon_{e}\right\}}=0$. 
With regard to the support of $\left(\widehat{a}_{n}, \widehat{u}_{n}\right)$, let $K_{n}$ be the compact support of $u_{n}$. Then

$$
\begin{aligned}
\operatorname{supp}\left(\widehat{a}_{n}, \widehat{u}_{n}\right) & =\overline{\left\{\left(\phi, \epsilon_{e}\right): \phi\left(a_{n}\right)+u_{n}(e) \neq 0\right\}} \cup \overline{\left\{\left(0, \epsilon_{x}\right): u_{n}(x) \neq 0\right\}} \\
& \subset \overline{\left\{\left(\phi, \epsilon_{e}\right): \phi\left(a_{n}\right)+u_{0}(e) \neq 0\right\}} \cup K_{n}^{\prime} \\
& =\overline{\left\{\phi \in \sigma(A): \phi\left(a_{n}\right) \neq-u_{0}(e)\right\} \times\left\{\epsilon_{e}\right\}} \cup K_{n}^{\prime} .
\end{aligned}
$$

But

$$
L_{n}=\overline{\left\{\phi \in \sigma(A): \widehat{a}_{n}(\phi) \neq-u_{0}(e)\right\}}
$$

is a closed subset of the compact support of $\widehat{a}_{n}$, and hence is compact. Since

$$
\left\{\phi \in \sigma(A): \phi\left(a_{n}\right) \neq-u_{0}(e)\right\} \times\left\{\epsilon_{e}\right\} \subset L_{n} \times\left\{\epsilon_{e}\right\}
$$

the compactness of the support of $\left(\widehat{a}_{n}, \widehat{u}_{n}\right)$ follows.

It remains to show that $\left(\phi_{0}, \epsilon_{e}\right) \notin \operatorname{supp}\left(\widehat{a}_{n}, \widehat{u}_{n}\right)$. It suffices to show that $\phi_{0} \notin L_{n}$. If $\phi_{0} \in L_{n}$ there exists a net $\phi_{\alpha}$ such that $\phi_{\alpha} \rightarrow \phi_{0}$ and $\phi_{\alpha}\left(a_{n}\right) \neq$ $-u_{0}(e)$ for all $\alpha$. But as $W_{n}$ is a neighborhood of $\phi_{0}, \phi_{\alpha}$ is eventually in $W_{n}$ and hence for sufficiently large $\alpha$,

$$
\widehat{a}_{n}\left(\phi_{\alpha}\right)=-u_{0}(e), \quad \text { i.e. } \quad \phi_{\alpha}\left(a_{n}\right)=-u_{0}(e) .
$$

This is of course a contradiction.

(ii) Let $\phi_{0} \in \sigma(A)$ and $\widehat{a}_{0} \in k\left(\left\{\phi_{0}\right\}\right)$. We want to approximate $\widehat{a}_{0}$ by a net $\left(\widehat{a}_{\alpha}\right)$ in $k_{00}\left(\left\{\phi_{0}\right\}\right)$ (that is, each $\widehat{a}_{\alpha}$ must vanish on a neighborhood of $\phi_{0}-$ as $\sigma(A)$ is compact we do not need to require that $\widehat{a}_{\alpha}$ has compact support). By our assumption $\left\{\left(\phi_{0}, \epsilon_{e}\right)\right\}$ is a local spectral set and of course

$$
\left\langle\left(\widehat{a}_{0}, 0\right),\left(\phi_{0}, \epsilon_{e}\right)\right\rangle=\phi_{0}\left(a_{0}\right)=0 .
$$

Compactness of $E$ implies the compactness of the support of $\left(\widehat{a}_{0}, 0\right)$. Hence there exists a net $\left(\widehat{a}_{\alpha}, \widehat{u}_{\alpha}\right) \in k_{00}\left(\left\{\left(\phi_{0}, \epsilon_{e}\right)\right\}\right)$ such that

$$
\left\|\left(\widehat{a}_{0}, 0\right)-\left(\widehat{a}_{\alpha}, \widehat{u}_{\alpha}\right)\right\|=\left\|a_{0}-a_{\alpha}\right\|+\left\|u_{\alpha}\right\| \rightarrow 0 .
$$

Since sets like $U \times\left\{\epsilon_{e}\right\}$ where $U$ is a neighborhood of $\phi_{0}$ in $\sigma(A)$ form a neighborhood base at $\left(\phi_{0}, \epsilon_{e}\right)$ (Proposition 2.4), there exists one such set for which

$$
\left.\left(\widehat{a}_{\alpha}, \widehat{u}_{\alpha}\right)\right|_{U \times\left\{\epsilon_{e}\right\}}=0 \text { and hence }\left.\widehat{a}_{\alpha}\right|_{U}=-u_{\alpha}(e) .
$$

If $1_{A}$ is the identity of $A$, then $\left.\left(\widehat{a}_{\alpha}+u_{\alpha}(e) \widehat{1}_{A}\right)\right|_{U}=0$, and

$$
\left\|\widehat{a}_{\alpha}+u_{\alpha}(e) \widehat{1}_{A}-\widehat{a}_{0}\right\| \leq\left\|\widehat{a}_{\alpha}-\widehat{a}_{0}\right\|+\left|u_{\alpha}(e)\right|\left\|\widehat{1}_{A}\right\| \rightarrow 0 .
$$

Thus $\left\{\phi_{0}\right\}$ is a spectral set.

Our next theorem concerns primary ideals in $A \times{ }_{\theta} A(G)$. For this we need a couple of preliminary results concerning the support of linear functionals on regular Banach algebras of functions. The notion of support in this generality was defined by Herz in [12, p. 101]. But Herz does not indicate the 
properties that we require here. For this reason we mention the following two results.

3.7. Lemma. Let $A$ be a regular Banach algebra in $\mathcal{C}_{0}(X)$ where $X$ is the spectrum of $A$. Let $T \in A^{\prime}$ and $a \in X$. Then the following assertions are equivalent:

(i) The linear functional $\epsilon_{a}: A \rightarrow \mathbb{C}, \epsilon_{a}(v)=v(a)$, is the weak $k^{*}$ limit of operators $v \cdot T$ where $v \in A$ (the product being the module product).

(ii) If $u \in A$ and if $u \cdot T=0$, then $u(a)=0$.

(iii) For every neighborhood $V$ of a there exists a function $v \in A$ with $\operatorname{supp} v \subset V$ and $\langle T, v\rangle \neq 0$.

Proof. The proof of Eymard for $A=A(G)$ in [8, Proposition 4.4, p. 225] can be applied with natural modifications.

For $A$ as in the above lemma, the support of an element $T \in A^{\prime}$ is defined as the set of all $a \in X$ satisfying the equivalent conditions of the lemma.

3.8. Lemma. Let $A$ be a regular Banach algebra in $\mathcal{C}_{0}(X)$ where $X$ is the spectrum of $A$, and suppose that elements with compact support are dense in $A$. If a singleton $\left\{x_{0}\right\} \subset X$ is a local spectral set and if $T \in A^{\prime}$ is such that $\operatorname{supp} T=\left\{x_{0}\right\}$, then $T=\lambda \epsilon_{x_{0}}$ for some $\lambda \in \mathbb{C}$.

This result for the case of $A=A(G)$ was proved in [8, Lemma 3.8, p. 221 and Proposition 4.8(6), p. 226]. Eymard uses the group structure of $G$ and so his proof does not seem to carry over to our general case. In our proof however we avoid any use of group structure, and instead use a hint in [12, p. 101] and Lemma 3.7 .

Proof. We divide the proof into several steps. The idea in Steps 1-3 is to show that

$$
v \cdot T=v\left(x_{0}\right) T \quad \text { for all } v \in A .
$$

STEP 1. If $u, v \in A \cap \mathcal{C}_{00}(X)$ and if $u=v$ on a compact neighborhood of $x_{0}$, then $\langle T, u\rangle=\langle T, v\rangle$.

Note that $x_{0} \notin K=\operatorname{supp}(u-v)$. So for every $x \in K$ there exists a neighborhood $U_{x}$ such that for every $w \in A$ with $\operatorname{supp} w \subset U_{x}$ we have $\langle T, w\rangle=0$. Let $V_{x}$ be a compact neighborhood of $x$ such that $V_{x} \subset U_{x}$. From compactness of $K$, there exist $x_{1}, \ldots, x_{n} \in K$ such that $K \subset \bigcup_{j=1}^{n} V_{x_{j}}$. By [13, Theorem 39.21, p. 495] the restricted algebra $A_{K}=\left\{\left.u\right|_{K}: u \in A\right\}$ is a normal Banach algebra of functions on the compact space $K$, and therefore by a partition of unity, there exist $v_{1}, \ldots, v_{n}$ in $A$ such that

$$
\operatorname{supp}\left(\left.v_{j}\right|_{K}\right) \subset V_{x_{j}} \cap K,\left.\quad \sum_{j=1}^{n} v_{j}\right|_{K}=1 .
$$


Using regularity of $A$, let $w_{j} \in A$ be such $\left.w_{j}\right|_{V_{x_{j}}}=1$ and $\operatorname{supp} w_{j} \subset U_{x_{j}}$. Let $u_{j}=v_{j} w_{j}$ for $j=1, \ldots, n$. Then

$$
\operatorname{supp} u_{j} \subset U_{j},\left.\quad \sum_{j=1}^{n} u_{j}\right|_{K}=1 .
$$

Since $\left\langle T,(u-v) u_{j}\right\rangle=0$, we get

$$
\langle T, u-v\rangle=\sum_{j=1}^{n}\left\langle T,(u-v) u_{j}\right\rangle=0,
$$

as we wanted to show.

Step 2. If $v \in A \cap \mathcal{C}_{00}(X)$ and if $v\left(x_{0}\right)=0$ then $v \cdot T=0$.

Since $\left\{x_{0}\right\}$ is a local spectral set, $v$ is the norm limit of a sequence $v_{n} \in v \in A \cap \mathcal{C}_{00}(X)$ such that $\operatorname{supp} v_{n}$ is disjoint from $\left\{x_{0}\right\}$. Then by Step $1, v_{n} \cdot T=0$ for all $n$ and hence $v \cdot T=0$.

SteP 3. If $v \in A \cap \mathcal{C}_{00}(X)$, then $v \cdot T=v\left(x_{0}\right) T$.

Let $v_{0} \in A \cap \mathcal{C}_{00}(X)$ be such that $v_{0}=1$ on a neighborhood of $x_{0}$. Then by Step $1, v_{0} \cdot T=T$. On the other hand, by Step $2,\left(v-v\left(x_{0}\right) v_{0}\right) \cdot T=0$ and hence $v \cdot T=v\left(x_{0}\right) v_{0} \cdot T=T$.

Now we can easily prove (19) by using the fact that the elements with compact support are dense in $A$. The proof of the lemma can now be completed as follows: since $\operatorname{supp} T=\left\{x_{0}\right\}$, by Lemma 3.7 there exists a net $\left(v_{\alpha}\right)$ in $A$ such that $\epsilon_{x_{0}}=$ weak $^{*}-\lim _{\alpha} v_{\alpha} \cdot T$. Thus for every $u \in A$,

$$
u\left(x_{0}\right)=\left\langle\epsilon_{x_{0}}, u\right\rangle=\lim _{\alpha}\left\langle v_{\alpha} \cdot T, u\right\rangle=\lim _{\alpha}\left\langle v_{\alpha}\left(x_{0}\right) T, u\right\rangle=\mu\langle T, u\rangle,
$$

where $\mu=\lim _{\alpha} v_{\alpha}\left(x_{0}\right)$. As $\mu \neq 0$, we can set $\lambda=1 / \mu$.

The following is a generalization of a famous result of Eymard [8, Corollary 1, p. 229]. The proof of Eymard now carries over.

3.9. Theorem. Let $A$ be a regular Banach algebra in $\mathcal{C}_{0}(X)$ where $X$ is the spectrum of $A$ and suppose that the elements with compact supports are dense in A. Suppose that every singleton $\{x\}$ is a local spectral set for $A$. Then every closed primary ideal of $A$ is a maximal modular ideal in $A$.

As a corollary of the above theorem and Theorem 3.5 we obtain:

3.10. Corollary. Let A be a commutative, semisimple, regular Banach algebra with identity. Suppose that every singleton $\{\phi\} \subset \sigma(A)$ is a spectral set for $A$. Then every closed primary ideal in $A \times_{\theta} A(G)$ is a maximal modular ideal. 


\section{References}

[1] W. G. Bade, H. G. Dales, and Z. A. Lykova, Algebraic and strong splittings of extensions of Banach algebras, Mem. Amer. Math. Soc. 656 (1999).

[2] B. A. Barnes, The role of minimal idempotents in the representation theory of locally compact groups, Proc. Edinburgh Math. Soc. 23 (1980), 229-238.

[3] F. F. Bonsall and J. Duncan, Complete Normed Algebras, Springer, New York, 1973.

[4] H. G. Dales, Banach Algebras and Automatic Continuity, Oxford Univ. Press, Oxford, 2000.

[5] H. G. Dales and A. T.-M. Lau, The second duals of Beurling algebras, Mem. Amer. Math. Soc. 836 (2005).

[6] A. Derighetti, Quelques observations concernant les ensembles de Ditkin d'un groupe localement compact, Monatsh. Math. 101 (1986), 95-113.

[7] A. Derighetti, M. Filali, and M. Sangani-Monfared, On the ideal structure of some Banach algebras related to convolution operators on $L^{p}(G)$, J. Funct. Anal. 215 (2004), 341-365.

[8] P. Eymard, L'algèbre de Fourier d'un groupe localement compact, Bull. Soc. Math. France 92 (1964), 181-236.

[9] —, Algèbres $A_{p}$ et convoluteurs de $L^{p}$, in: Lecture Notes in Math. 180, Springer, New York, 1971, 364-381.

[10] B. Forrest, Amenability and the structure of the algebras $A_{p}(G)$, Trans. Amer. Math. Soc. 343 (1994), 233-243.

[11] N. Grønbæk, Weak and cyclic amenability for non-commutative Banach algebras, Proc. Edinburgh Math. Soc. 35 (1992), 315-328.

[12] C. Herz, Harmonic synthesis for subgroups, Ann. Inst. Fourier (Grenoble) 23 (1973), no. $3,91-123$.

[13] E. Hewitt and K. A. Ross, Abstract Harmonic Analysis, Vol. 2, Springer, New York, 1970.

[14] B. E. Johnson, Cohomology in Banach algebras, Mem. Amer. Math. Soc. 127 (1972).

[15] B. E. Johnson and M. C. White, A non-weakly amenable augmentation ideal, preprint.

[16] E. Kaniuth and A. T.-M. Lau, Spectral synthesis for $A(G)$ and subspaces of $V N(G)$, Proc. Amer. Math. Soc. 129 (2001), 3253-3263.

[17] J. L. Kelley and I. Namioka, Linear Topological Spaces, Springer, New York, 1963.

[18] A. T.-M. Lau, Analysis on a class of Banach algebras with applications to harmonic analysis on locally compact groups and semigroups, Fund. Math. 118 (1983), 161175 .

[19] A. T.-M. Lau and R. Loy, Weak amenability of Banach algebras on locally compact groups, J. Funct. Anal. 145 (1997), 175-204.

[20] V. Runde, Lectures on Amenability, Springer, New York, 2002.

[21] M. Sangani-Monfared, Character amenability of Banach algebras, preprint.

[22] —, Extensions and isomorphims for the generalized Fourier algebras of a locally compact group, J. Funct. Anal. 198 (2003), 413-444.

Department of Mathematics and Statistics

University of Windsor

Windsor, ON, N9B 3P4, Canada

E-mail: monfared@uwindsor.ca

Received May 25, 2006

Revised version October 26, 2006 